\title{
GASTROSTOMIAS E FUNDOPLICATURAS: ESTUDO RETROSPECTIVO DE 5 ANOS EM PACIENTES PEDIÁTRICOS NO HOSPITAL MUNICIPAL JESUS/ RJ
}

\author{
GASTROSTOMY AND FUNDOPLICATION: A 5-YEAR RETROSPECTIVE STUDY \\ AT PEDIATRIC PATIENTS IN HOSPITAL MUNICIPAL JESUS/ RJ
}

\author{
Lisieux Eyer de Jesus - TCBC-RJ ${ }^{1}$ \\ Paulo César Costa Monteiro ${ }^{2}$ \\ Renata Restay Siqueira ${ }^{3}$ \\ Elisiane Bezerra Marinho ${ }^{3}$ \\ Pedro Augusto Vital Nogueira ${ }^{4}$
}

\begin{abstract}
RESUMO: Objetivo: Analisar o quadro clínico, morbi-mortalidade e resultados da fundoplicatura gastroesofágica em crianças, com ênfase em portadores de encefalopatias crônicas. Método: Foram estudados retrospectivamente os prontuários de 55 pacientes em série, submetidos a fundoplicaturas e/ou gastrostomias por 5 anos (1994-1999), analisando manifestações clínicas, características epidemiológicas e evolução pósoperatória. Análises estatísticas, quando pertinentes, utilizaram o método do qui-quadrado. Resultados: Manifestações respiratórias, pacientes com menos de seis meses e encefalopatas predominaram. Opistótono esteve relacionado a formas graves. A mortalidade até 30 dias foi de 7,3\%, significativamente maior em cardiopatas congênitos. Complicações imediatas da cirurgia foram basicamente atelectasias e pneumonias $(14,6 \%)$, relacionadas estatisticamente à desnutrição grave, e infecções da ferida $(5,5 \%)$. O índice de recorrência de Doença do Refluxo Gastro-Esofagiano foi de 14,5\%. O índice de pneumonias caiu de 65,5\% em pré-operatório para 16,5\% em pós-operatório tardio. Em média houve melhora nutricional a longo prazo, embora com ampla variação individual. Conclusão: É essencial manter um alto índice de suspeita para Doença do Refluxo Gastro-Esofagiano em lactentes e crianças encefalopatas com manifestações respiratórias. Nestes grupos a indicação cirúrgica é mais freqüente e bastante segura, exceto em presença de desnutrição grave e cardiopatia congênita. Opistótono é marcador de doença grave. Os resultados da cirurgia a longo prazo são favoráveis em crianças encefalopatas.
\end{abstract}

Descritores: Refluxo gastroesofágico; Dano cerebral crônico; Fundoplicatura; Gastrostomia.

\section{INTRODUÇÃO}

O tratamento operatório da doença do refluxo gastroesofágico (DRGE), apesar de constituir uma das cirurgias mais comuns em pediatria é palco de diversas controvérsias, especialmente frente à necessidade de realizar gastrostomia alimentar em encefalopatas

1. Cirurgiã pediátrica do Hospital Municipal Jesus e Hospital Universitário Antônio Pedro, UFF; Supervisora de Residência Médica em cirurgia pediátrica do Hospital Municipal Jesus.

2. Cirurgião pediátrico do Hospital Municipal Jesus e Hospital Universitário Antônio Pedro, UFF.

3. Médicas residentes do $2^{\circ}$ ano em Cirurgia Pediátrica do Hospital Municipal Jesus.

4. Cirurgião pediátrico do Hospital Municipal Jesus e Hospital da Lagoa; Chefe do Serviço de Cirurgia Pediátrica do Hospital Municipal Jesus.

Recebido em: 05/06/2001

Aceito para publicação em: 10/06/2002

Trabalho realizado no Serviço de Cirurgia Pediátrica do Hospital Municipal Jesus, Rio de Janeiro, RJ, Brasil. 
crônicos com ou sem DRGE clinicamente detectável. A fundoplicatura gastro-esofágica (FP) na criança se aplica, para vários autores, entre os quais nos incluímos, como procedimento acessório à realização de gastrostomia (GT) alimentar, especialmente em encefalopatas (EP) com distúrbio de deglutição. Neste grupo, freqüentemente, a presença ou não de DRGE é omitida ou desconsiderada, e as implicações da cirurgia nesta porção da amostragem - geralmente majoritária - tem a avaliação dificultada. Em face da possibilidade de doença respiratória por DRGE e/ou aspiração de conteúdo faríngeo há discussões quanto ao potencial de melhora das condições respiratórias, e, em decorrência, tempo de sobrevida ou qualidade de vida do paciente EP após a cirurgia de FP com GT, já que esta não elimina a possibilidade de aspiração a partir da cavidade oral.

Pretendemos aqui mostrar a experiência de cinco anos com a cirurgia de FP em nosso serviço e discutir as particularidades com relação a vários subgrupos de crianças, notadamente EP e lactentes de baixa idade, considerando para análise, inclusive, as particularidades sócio-econômicas que interagem como definidoras de conduta para nossa populaçãoalvo.

\section{MÉTODO}

Estudo retrospectivo de 55 pacientes menores de 12 anos de idade nos quais foram indicadas fundoplicaturas gastro-esofágicas (FP) acompanhadas ou não de gastrostomias (GT) pela equipe de Cirurgia Pediátrica do Hospital Municipal Jesus (HMJ), um hospital pediátrico terciário, referência para neurocirurgia infantil no Município do Rio de Janeiro, no período de 1994 a 1999. Neste período foram realizadas 65 cirurgias de FP e/ou GT (média de 13/ ano). Foram excluídos 10 pacientes devido a problemas logísticos de acesso ao prontuário médico.

Testes estatísticos, quando necessários, foram feitos através do método do qui-quadrado. Os responsáveis pelos pacientes receberam informações detalhadas quanto à indicação, objetivos e riscos dos procedimentos e autorizaram previamente as cirurgias, para todas as crianças.

\section{RESULTADOS}

Durante o período do estudo, foram operados 55 pacientes (60\% sexo masculino) entre dois meses e 10 anos de idade (mediana seis meses e média 16,9 meses), com 29 crianças menores que seis meses de idade $(43,6 \%)$ e 34 até um ano de idade $(52,7 \%)$. Vinte e oito $(50,8 \%$ ) crianças foram operadas durante a primeira internação hospitalar (14 bebês transferidos da maternidade onde ocorreu seu nascimento, presumindo complicações perinatais graves e/ou defeitos congênitos severos). Sete pacientes $(12,7 \%)$ apresentavam apenas uma internação anterior e os demais $(36,4 \%)$ tinham história de duas ou mais internações, numa distribuição bimodal entre crianças na evolução de sua primeira internação e crianças crônica e repetitivamente hospitalizadas (Gráfico-1).

Os tempos médios de internação foram de 43 dias em pré-operatório e 19 dias em pós-operatório, excluídos dois pacientes com internações maiores que 300 dias por problemas sociais. Verificamos $85,5 \%$ de EP, 3,6\% de portadores de broncodisplasia pulmonar (todos EP) e 9,1\% de cardiopatas congênitos. Apenas oito pacientes não apresentavam problemas neurológicos $(14,6 \%)$, inclusive um portador de atresia de esôfago e uma criança com estenose faríngea congênita (Tabela-1). A maioria dos EP foi encaminhada para realização de gastrostomia alimentar ou por problemas respiratórios crônicos atribuídos à aspiração orofaríngea e distúrbios de deglutição. Raramente havia diagnóstico ou suspeita clínica de DRGE em EP, cujas etiologias eram lesão hipóxico-isquêmica perinatal $(28,6 \%)$, síndromes genéticas $(21,4 \%)$, hidrocefalia $(7,1 \%)$ e seqüela de hemorragia intracraniana ligada a prematuridade $(7,1 \%)$. Nos demais EP não havia indicação quanto à etiologia da neuropatia de base nos prontuários.

Gráfico 1 - Frequência das internações.

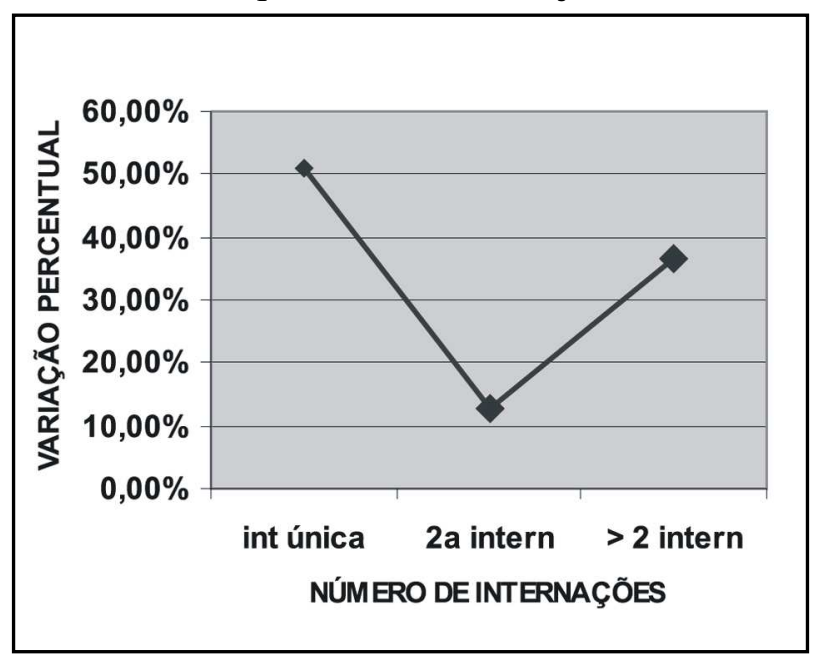


Tabela 1 - Diagnósticos de base.

\begin{tabular}{lrr}
\hline Diagnóstico Associado & N & \% \\
\hline Encefalopatas & 47 & 85,5 \\
Síndrome Genética & 12 & 21,8 \\
Cardiopatia Congênita & 5 & 9,1 \\
Prematuridade & 14 & 29,8 \\
Broncodisplásicos & 2 & 3,6 \\
Sem Doença Neurológica & 8 & 14,6 \\
Atresia de Esôfago & 1 & 1,8 \\
Estenose Faringe & 1 & 1,8 \\
\hline
\end{tabular}

Os achados clínicos por faixa etária e em EP são mostrados na Tabela-2. Quarenta pacientes, todos $\mathrm{EP}$, recebiam nutrição por gavagem $(85,1 \%$ dos $\mathrm{EP})$. Todos os pacientes tiveram solicitado um estudo contrastado de trato digestivo superior (ECTDS), buscando não somente o diagnóstico da DRGE como também verificar complicações, doenças ou particularidades anátomo-funcionais associadas. Reservamos exames endoscópicos digestivos para pacientes em curso de complicações (hemorragia digestiva ou estenose de esôfago). Exames para estudo do aparelho respiratório foram exceção, para pacientes com complicações respiratórias específicas ou dúvida quanto ao papel de DRGE para a gênese dos sintomas.

Todos os pacientes foram submetidos a tratamento clínico por períodos variáveis, não inferiores a quatro semanas, com medidas posturais (100\% dos casos) e pró-motílicos (82\%). Bloqueadores histamínicos ou antiácidos foram usados em 10 casos $(18,2 \%)$. A indicação de cirurgia se deu pela ausência de resposta ao tratamento clínico ou, principalmente, pela necessidade de gastrostomia alimentar determinada por variáveis sócio-familiares, necessidade de reposição nutricional agressiva e/ou pela impossibilidade do especialista em fonoaudiologia em tratar o distúrbio de deglutição.

Quarenta e nove pacientes $(89,1 \%)$ receberam FP (83,6\% pela técnica de Nissen e 5,5\% FP parcial). Em dois casos já havia gastrostomia alimentar prévia, após a qual surgiram sintomas sugestivos de DRGE. Quarenta e cinco ( 81,9\%) pacientes foram gastrostomizados (100\% dos EP). A realização de FP nos portadores de gastrostomia é rotina em nosso serviço, independente do reconhecimento de DRGE. Excepcionalmente quatro crianças $(7,3 \%)$ receberam apenas gastrostomias, fundamentalmente pelo alto risco anestésico. Três $(5,5 \%)$ crianças receberam alguma forma de drenagem pilórica e em três casos $(5,5 \%)$ foram indicadas traqueostomias por paralisia de cordas vocais.

Houve 12 óbitos (21.8\%), oito após 30 dias pós-operatórios. Apenas quatro pacientes faleceram antes de 30 dias pós-operatórios, dois na $1^{\text {a }}$ semana, por complicações do ato anestésico. A mortalidade cirúrgica foi de 7,3\% e a tardia de $14,6 \%$. Foi possível seguimento por mais de seis meses pós-operatórios em $51,1 \%$ dos casos $(n=22)$, excluídos os óbitos. Em 23,3\% dos pacientes $(\mathrm{n}=10)$ não houve seguimento do paciente em nosso hospital após a alta. $\mathrm{O}$ seguimento dos pacientes foi basicamente clínico: exames complementares foram reservados a casos que apresentavam sintomas.

Não foi verificada diferença estatística quanto à freqüência de internações hospitalares préoperatórias entre EP e crianças sem distúrbios neurológicos (NN )portadoras de DRGE ( $\mathrm{p}>0,05)$, apesar da tendência a internações repetitivas em pacientes NN com relação aos EP (respectivamente $25 \%$ e $55,3 \%$ de internação única).

Foi possível traçar comparações quanto ao quadro clínico de apresentação para EPe NN (Tabela2). A incidência de manifestações respiratórias da DRGE (pneumonia única ou repetitiva ou crises apneicas), a suspeita de DRGE apenas por sintomas digestivos típicos (vômitos e regurgitação freqüentes e repetitivos), postura em opistótono e o índice de complicações pós-operatórias não apresentaram diferenças estatísticas entre NN e EP. No grupo de EP a incidência de distúrbios de deglutição foi maior que entre pacientes $\mathrm{NN}(\mathrm{p}<0,05)$, mas não exclusiva. O diagnóstico clínico de desnutrição foi feito mais freqüentemente para $\mathrm{NN}(\mathrm{p}<0,01)$, embora quando submetidos a um critério matemático (número de casos com peso $\geq$ percentil 10$)^{1}$ não houvesse diferença detectável (20\% dos pacientes EP acima do percentil 10 de peso versus $25 \%$ dos NN).

Houve nítidas diferenças nas manifestações clínicas da DRGE entre crianças até seis meses de idade e crianças mais velhas: a incidência de pneumonias foi maior a partir do segundo semestre ( $p<0,01)$, assim como desnutrição $(p<0,01)$, distúrbio de deglutição $(p<0,01)$ e diagnóstico de DRGE por vômitos recorrentes e regurgitação alimentar $(p<0,05)$ (Tabela-2). É perceptível uma relação entre 
Tabela 2 - Dados clínicos.

\begin{tabular}{|c|c|c|c|c|c|}
\hline Problema & Total (N/\%) & $\operatorname{EP}(\mathbf{N} / \%)$ & $\mathbf{N N}(\mathbf{N} / \%)$ & $\leq 6$ meses $(\mathrm{N} / \%)$ & $>6$ meses $(\mathrm{N} / \%)$ \\
\hline Pneumonias & $36 \quad(65,4)$ & $30(63,8)$ & $6(75)$ & $12(41,4)$ & $24 \quad(92,3)$ \\
\hline Pneumonias repetição & $24 \quad(43,6)$ & $19(40,4)$ & $5(62,5)$ & $7 \quad(24,1)$ & $17 \quad(65,4)$ \\
\hline Crise apnéia & $8(14,6)$ & $7(14,9)$ & $1(12,5)$ & $6 \quad(20,7)$ & $2 \quad(7,7)$ \\
\hline Distúrbio deglutição & $32(58,2)$ & $30(63,8)$ & $2(25)$ & $12(41,4)$ & $20 \quad(76,9)$ \\
\hline Vômitos e regurgitação & $9(16,3)$ & $7(14,9)$ & $2(25)$ & $2 \quad(6,9)$ & $8 \quad(26,9)$ \\
\hline Hemorragia digestiva & $5 \quad(9,1)$ & $5(9,1)$ & ZERO & $1 \quad(3,4)$ & $4 \quad(15,4)$ \\
\hline Opistótono & $5 \quad(9,1)$ & $4(8)$ & $1(12,5)$ & $3 \quad(10,3)$ & $3(11,5)$ \\
\hline Desnutrição "clínica" & $25 \quad(45,5)$ & $18(38,3)$ & $7(87,5)$ & $7 \quad(24,1)$ & $18 \quad(69,2)$ \\
\hline
\end{tabular}

internações repetidas e faixa etária (média de 11,4 meses para internações únicas e 29,5 meses para internações repetitivas).

Resultados do ECTDS puderam ser resgatados para 39 pacientes. O exame foi normal ou inconclusivo em 10\%, documentou distúrbio de deglutição em 17 casos $(43,6 \%)$ e DRGE em 22 doentes (56,4\%). Cinco pacientes mostraram distúrbio motor do esôfago $(12,8 \%)$ e três distúrbio de esvaziamento gástrico $(7,7 \%)$. Em apenas dois havia DRGE associada a um cárdia permanentemente aberto (RGE grau V) ${ }^{2}$. Dois pacientes $(5,1 \%)$ apresentavam estenose péptica do esôfago e houve quatro casos de broncoaspiração durante o exame (10,3\%), sem consequiências clínicas imediatas ou tardias detectáveis. Quatro pacientes $(10,6 \%)$ apresentavam hérnia de hiato ou alterações anatômicas do diafragma. Quatro foram submetidos a endoscopia digestiva, detectando três casos de esofagite, três casos de incompetência do cárdia, um esôfago de Barrett e um portador de estenose péptica do esôfago.

Houve apenas um sobrevivente após 30 dias pós operatórios entre pacientes submetidos exclusivamente a GT (50\% de óbito na $1^{\text {a }}$ semana e $75 \%$ no primeiro mês). Nenhum caso de óbito com menos de 30 dias ocorreu em paciente com DRGE como único diagnóstico nosológico. A mortalidade foi significativamente maior $(p<0,01)$ entre cardiopatas e em pacientes transferidos da maternidade $(p<0,05)$, mas não foi pior em EP com relação aos NN ( $>>0,05)$ ou entre lactentes com menos de meses e crianças mais velhas $(p>0,05)$. Houve diferença estatisticamente significativa quanto aos índices de mortalidade também entre crianças com desnutrição grave (peso abaixo do percentil 10 para a idade) $(\mathrm{p}<0,01)$ e entre crianças que apresentavam opistótono $(p<0,05)$. Não houve diferenças significativas com relação à história pregressa de pneumonia, crise apneica ou distúrbio de deglutição.

Em pós-operatório precoce (30 dias) ocorreram oito complicações respiratórias (pneumonia ou atelectasia), três infecções da ferida operatória (duas eviscerações), um caso de disfagia e um de lesão esplênica (esplenectomia). Desnutrição protéico-calórica foi estatisticamente relacionada a complicações precoces em geral $(\mathrm{p}<0,01)$ e complicações respiratórias no pós-operatório imediato $(\mathrm{p}<0,05)$, porém não pôde ser relacionada à presença de infecções da ferida cirúrgica ou eviscerações.

Houve complicações precoces ou tardias em 19 pacientes $(36,4 \%)$, geralmente respiratórias, sem diferenças estatísticas significativas quanto à faixa etária ou EP de base. Ocorreram oito $(14,5 \%)$ recorrências de DRGE reconhecidas em bases clínicas, $50 \%$ até seis meses de pós-operatório, nove $(16,4 \%)$ de pneumonia pós-operatória não relacionadas obrigatoriamente à recidiva da DRGE, duas $(3,6 \%)$ obstruções intestinais por aderências (uma em pósoperatório precoce, ambos tratados clinicamente), duas $(3,6 \%)$ fístulas persistentes após retirada de GT, uma hérnia incisional $(1,8 \%)$ e uma $(1,8 \%)$ recorrência de hérnia de hiato sem DRGE. Notadamente o índice de pneumonias tardias - $16,4 \%$ - foi muito menor que o número de pneumonias no préoperatório - $65,5 \%$. O ganho ponderal no pósoperatório tendeu para uma melhora nutricional, com ganho médio de $19,1 \%$ de peso até 30 dias de pósoperatório e de $16,1 \%$ de peso além de seis meses, porém existiram variações amplas neste critério $(0 \mathrm{a}$ $63 \%$ até 30 dias e 48,6 a $130 \%$ de variação ponderal além de seis meses), possivelmente relacionáveis à condição clínica geral de cada paciente. 


\section{DISCUSSÃO}

As indicações para FP e tratamento de DRGE em pediatria, em especial em EP, são controversas em vários aspectos: é necessário adicionar $\mathrm{FP}$ em pacientes operados para confecção de GT alimentar? Qual a freqüência e expressão de DRGE nos EP? As pneumonias de repetição em EP são ligadas à DRGE ou à aspiração orofaríngea? Ocorre melhora respiratória, no tempo de sobrevida ou qualidade de vida do EP após FP com GT? O risco cirúrgico em EP é maior? É válido operar pacientes no primeiro semestre de vida em quem ainda é esperada a maturação dos mecanismos de contenção da junção esôfago-gástrica (JEG)? O que é DRGE ou regurgitação por imaturidade em crianças de baixa idade, em especial se EP?

A obtenção de respostas definitivas é dificultada pela predominância absoluta de trabalhos retrospectivos sem grupo controle e com definições não uniformes de DRGE, sem uma estratificação quanto à gravidade da neuropatia de base. No Brasil dificuldades logísticas para obter exames complementares dificultam o diagnóstico e existe uma premência de indicar GT em pacientes com distúrbios de deglutição numa população carente de instrução formal e instalações domiciliares satisfatórias. A disponibilidade de apoio clínico e social residencial é mínima e o índice de crianças EP abandonadas e institucionalizadas é elevado. Há problemas éticos quanto à validade de operar pacientes cujo prognóstico de sobrevida e/ou qualidade de vida é limitado e piorado pela pouca disponibilidade de fisioterapia e educação especial. Finalmente, tão fundamental quanto considerações técnicas é a qualidade de vida das famílias obtida pela facilidade para alimentar crianças EP provida pela GT e menor frequiência de internações hospitalares após a cirurgia, verificada para nossos pacientes e corroborada por outros autores ${ }^{3,4}$.

Em nosso estudo, a predominância de EP poderia ser explicada por se tratar de serviço de referência para crianças com lesões neurológicas, porém um alto índice de EP é a regra em estudos de tratamento cirúrgico da DRGE pediátrica. Os trabalhos mostram uma incidência pequena em crianças normais ${ }^{5}$.

A incidência máxima da cirurgia é no primeiro semestre de vida, em geral por problemas respiratórios, mormente as discussões quanto à conveniência de aguardar a eventual maturação do mecanismo de contenção da JEG, que no momento se acredita ocorrer aos quatro meses de idade ${ }^{6}$. Em nosso estudo as indicações precoces de cirurgia se devem também à necessidade de permitir a alta hospitalar para EP graves incapazes de nutrição oral. No grupo mais velho de pacientes predominam EP, porém há crianças normais, em quem percebemos um viés para indicação tardia.

Os tempos de internação pré e pós-operatória são semelhantes aos de outros autores (pré-operatório 19 a 43 dias e pós-operatório 14 a 26 dias) ${ }^{3,7}$, refletindo a gravidade dos pacientes e a necessidade de preparo respiratório para a anestesia. $\mathrm{O}$ grupo de cardiopatas congênitos demonstrou altíssima morbimortalidade. Estes dados estão de acordo com Baum et al., que citam mortalidade cirúrgica geral até 3,5 vezes maior para cardiopatas congênitos no primeiro ano de vida com relação à população controle ${ }^{8}$.

Sintomas respiratórios são importantíssimos na suspeita de DRGE embora erroneamente muitos médicos aguardem sintomas digestivos para o diagnóstico. A especificidade de vômitos/regurgitação é muito pequena, por sua alta freqüência em lactentes normais: $23 \%$ das crianças aos cinco meses de idade apresentam mais de três episódios de regurgitação alimentar por dia ${ }^{9}$. Tais manifestações são ainda mais frequientes em EP e raramente valorizadas, embora vários autores sublinhem a alta incidência de DRGE nestes casos: $68 \%$ a $83 \%$ dos EP com vômitos recorrentes têm DRGE ${ }^{10-12}$. Os demais sintomas digestivos de DRGE expressam complicações (hemorragia digestiva e disfagia) e são tardios. Anemia secundária à perda de sangue oculto por esofagite é muito valorizada ${ }^{10,11,13}$, mas pouco discriminatória em nosso meio pela alta frequência de anemia carencial. Pneumonias, crises de apnéia e dificuldades de manejo respiratório em portadores de broncodisplasia são as principais manifestações no primeiro semestre de vida, e infecções de vias aéreas persistem em todas as faixas etárias como expressão - por vezes única - da doença. Pneumonias de repetição são causadas por DRGE em até $5 \%$ dos casos em NN e têm indicado a pesquisa da doença ${ }^{14}$. Foi surpreendente perceber que os dados clínicos se assemelham para pacientes EP, freqüentemente encaminhados para GT sem a suspeita clínica de DRGE e NN, exceto pela presença aumentada, porém não exclusiva, de distúrbios de deglutição no primeiro grupo: há casos de DRGE grave em NN com incoordenação de deglutição, revertida prontamente 
após a cirurgia. A postura em opistótono é citada por poucos autores ${ }^{15}$ como sinal de DRGE e em geral é atribuída à hipertonia comum nos EP. Em nossa opinião este sinal pode ser um marcador de DRGE grave em crianças restritas ao leito pela faixa etária (lactentes) ou doença associada (EP), equivalendo à síndrome de Sandifer, descrita em crianças mais velhas com postura ortostática predominante ${ }^{15}$.

Há diferenças indiscutíveis entre crianças com menos de seis meses e crianças mais velhas, aparentemente pela evolução temporal da doença (maior freqüência de pneumonias simples e de repetição e pior estado nutricional), que podem também estar relacionadas à evolução da doença neurológica de base em EP. A indicação cirúrgica precoce parece ser uma tendência, baseada na melhor identificação da DRGE, baixa morbidade e bons resultados da cirurgia ${ }^{7,16}$.

A não disponibilidade de $\mathrm{pHmetria} \mathrm{esofágica}$ (padrão ouro) para diagnóstico é uma limitação séria em nosso estudo. Reiteramos a baixa sensibilidade do exame de ECTDS, capaz de detectar apenas 56\% de DRGE em nossos pacientes, apesar da fácil execução e disponibilidade ambulatorial com pequena colaboração do paciente e capacidade de prover dados anatômicos relevantes ao cirurgião. Apesar da deficiência de estudos diagnósticos pré-operatórios de alta precisão nossos dados referentes à melhora dos pacientes EP após GT alimentar e fundoplicatura sugerem um papel terapêutico para a FP. Parte da melhora clínica pode ser atribuída à alimentação exclusiva através da GT, porém a resolução de problemas respiratórios, opistótono e hemorragia digestiva não é facilmente explicável apenas pela GT alimentar.

Apresentamos dois casos de DRGE após GT simples, motivando reoperação. Vários autores citam FP após GT simples (2 a 30\% dos casos de FP) e dados já clássicos mostram até $50 \%$ de refluxo gastroesofágico "novo" após GT, inclusive com óbitos por broncoaspiração ${ }^{14,17}$. Stephen ${ }^{18}$, num grupo de EP sem DRGE submetidos à GT simples, mostra uma incidência de $41 \%$ de DRGE, dos quais $77 \%$ precisaram posteriormente de FP. Tivemos poucos pacientes submetidos a GT simples, e a maioria absoluta destas crianças faleceu precocemente, de forma que não podemos oferecer dados quanto às eventuais conseqüências tardias do procedimento.

A associação de DRGE e EP é extremamente freqüente, em especial em para encefalopatia hipóxico-isquêmica, talvez por lesão do núcleo central do vago, capaz de alterar difusamente a motricidade do trato digestivo. A DRGE pode também estar relacionada à hipertonia ou síndromes convulsionais e alterações anatômicas do hiato em decorrência de escolioses e vícios posturais ${ }^{10-12}$. Lohmer ${ }^{12}$, em estudo sistemático de pHmetria em EP institucionalizados com QI $<50$ em várias faixas etárias detectou $52 \%$ de DRGE, com $11 \%$ de exames inconclusivos, independente da idade ou postura do paciente, sugerindo a independência ou ineficácia de mecanismos de maturação. DRGE foi mais freqüente em presença de escoliose (OR 2,6), encefalopatia hipóxico-isquêmica (OR 2,6), crise convulsivas recorrentes $(\mathrm{OR} 2,8)$ e $\mathrm{QI}<35(\mathrm{OR} 4,3)^{12}$. Em nossa experiência têm ocorrido alguns casos de paralisia de cordas vocais (três neste estudo) levando à traqueostomia, e achamos possível que tais lesões sejam também atribuíveis à lesão do vago, na forma de disfunção do nervo laríngeo recorrente.

A mortalidade até 30 dias predominou em cardiopatas e portadores de malformações graves. Excluindo estes grupos a mortalidade cirúrgica foi zero. A mortalidade tardia foi de $14,5 \%$ corroborando o mau prognóstico individual dos EP: em estudo europeu recente o prognóstico de sobrevida após oito anos em crianças EP institucionalizadas (43\% lesões geneticas ou perinatais) variou desde $2 / 3$ dos pacientes vegetativos e imóveis a $81 \%$ em crianças com mobilidade preservada ${ }^{19}$. Complicações precoces (18\% dos casos) praticamente se restringiram a problemas respiratórios (pneumonia e atelectasia) e infecções de ferida cirúrgica. Dados de literatura variam entre $5 \%$ e $50 \%$ de complicações precoces, em especial respiratórias ${ }^{11}$. A presença de complicações e problemas respiratórios pós-operatórios foi relacionada à desnutrição grave ${ }^{20}$, sugerindo o uso de nutrição transjejunal para preparo pré-operatório nos pacientes com depleção nutricional mais severa.

Há uma tendência ao ganho nutricional (média de 19,1\% até 30 dias e 16,1\% após seis meses de pós-operatório), embora as variações sejam amplas, em especial a longo prazo $(48,6 \mathrm{a}+130 \%$ após seis meses). Atribuímos as variações à(s) doença(s) de base e condições sociais. Respondendo à alegação de que a cirurgia seria ineficaz para proteger pacientes em quem o mecanismo de broncoaspiração através da cavidade oral permanece constante houve uma queda na incidência de pneumonias de $65,5 \%$ no período pré-operatório para $16,4 \%$ nos casos seguidos 
por mais de seis meses após a cirurgia. A recidiva da DRGE detectada através de sinais clínicos foi de $14,5 \%$, coincidindo com os índices de 6 a $24 \%$ em literatura ${ }^{4,19}$. Com relação aos EP em geral houve melhora do paciente e satisfação dos familiares com relação à cirurgia. Todos foram capazes de adaptação muito rápida à GT. Concluimos que é fundamental um limiar baixo para suspeita, investigação e tratamento de DRGE em presença de sintomas respiratórios em crianças, especialmente EP e portadores de atresia de esôfago, considerados com risco maior que $50 \%$ para a doença. Não verificamos piora do risco cirúrgico associado à lesão neurológica, porém a presença de cardiopatia congênita determina mau prognóstico e desnutrição grave aumenta a morbimortalidade. Também entre lactentes de $1^{\circ}$ semestre não houve problemas específicos decorrentes da cirurgia. Postura em opistótono é marcadora específica de formas graves de DRGE na criança. De forma geral a realização de $\mathrm{FPe}$ GT em crianças $\mathrm{NN}$ teve resultados favoráveis, em especial quanto a um menor índice pósoperatório de pneumonias.

\begin{abstract}
Background: To study clinical characteristics, mortality, morbidity and results of fundoplicature with or without gastrostomy in children, emphasizing neurologically impaired patients. Methods: We studied retrospectively medical documents of 55 successive patients operated on in a period of five years (1994-1999), analysing variables related to clinical manifestations, epidemiology and post-operative evolution. Statistical analysis, when applicable, were perfomed using qui-square method. Results: Respiratory symptoms, less than 6-month old infants and neurologically impaired children predominated. Opisthotonus was related to severe forms of gastroesophageal reflux disease (GERD). Thirty day mortality was 7,3\%, significantly worse for those with congenital cardiac disease. Precocious complications were basically atelectasis and pneumonia $(14,6 \%)$, with positive correlation to pre-operative severe malnutrition and surgical infections $(5,5 \%)$. GERD recurred in $14,5 \%$. After six post-operative months follow-up, cumulative taxes for pneumonias were $16,5 \%$, versus $65 \%$ pre-operatively. In general there was nutritional improvement, despite large individual variations. Conclusion: It is essential to maintain a high index of suspition for GERD in small infants and neurologically impaired children with respiratory symptoms. In these groups surgery is more frequently necessary and essentially safe, except for nutritionally depleted children and in presence of severe cardiac defects. Opisthotonus suggests severe GERD. Surgical results are very favourable even for neurologically impaired children.
\end{abstract}

Key words: Gastroesophageal reflux; Brain damage, chronic; Fundoplication; Gastrostomy.

\section{REFERÊNCIAS}

1. Vaughan VC, McKay RJ, Nelson WE. Tratado de pediatria. $7^{\text {a }}$ Edição. México. Salvat Mexicana de Ediciones, 1980, pp. 40-43.

2. McCauley RG, Darling DB, Leonidas JC, et al. Gastroesophageal reflux in infants and children: a useful classification and reliable physiologic technique for its demonstration. AJR Am J Roentgenol, 1978, 130(1): 47-50.
3. Rice H, Seashore JH, Touloukian, RJ. - Evaluation of Nissen fundoplication in neurologically impaired children. J Pediatr Surg, 1991, 26(6): 697-701.

4. Vane DW, Harmel RP, King DR, et al. - The effectiveness of Nissen fundoplicature in neurologically impaired children with gastroesophageal reflux. Surgery, 1985, 98(4): 662-667.

5. Snyder CL, Ramachandran V, Kennedy AP, et al. Efficaccy of partial wrap fundoplication for gastroesophageal reflux after repair of esophageal atresia. J Pediatr Surg, 1997, 32(7): 1089-1092. 
6. Boix-Ochoa J, Canals J. - Maturation of the lower esophagus. J Pediatr Surg, 1976, 11(5): 749-756.

7. Krishnamoorthy M, Mintz A, Liem, T, et al. - Diagnosis and treatment of respiratory symptoms of initially unsuspected gastroesophageal reflux in infants. Am Surg, 1994, 60(10): 783-785.

8. Baum VC, Barton DM, Gutgesell HP. - Influence of congenital heart disease on mortality after noncardiac surgery in hospitalized children. Pediatrics, 2000, 105(2): 332-335.

9. Nelson SP, Chen EH, Syniar GM, et al. - Prevalence of symptoms of gastroesophageal reflux during childhood: a pediatric practice-based survey. Pediatric Practice Research Group. Arch Pediatr Adolesc Med, 2000, 154(2): 150-154.

10. Sondheimer JM, Morris BA. - Gastroesophageal reflux among severely retarded children. J Pediatr, 1979, 94(5): 710-714.

11. Byrne WJ, Euler AR, Ashcraft E, et al. Gastroesophageal reflux in the severely retarded who vomit: criteria for and results of surgical intervention in twenty-two patients. Surgery, 1982, 91(1):95-98.

12. Lohmer CJM, Niezen de Boer MC, KlinkenbergKnol EC, et al. - The prevalence of gastroesopphageal reflux disease in institutionalized intellectually disabled individuals. Am J Gastroenterol, 1999, 94(3):804810 .

13. Wilkinson JD, Dudgeon DL, Sondheimer JM. - A comparison of medical and surgical treatment of gastroesophageal reflux in severely retarded children. J Pediatr, 1981, 99(2): 202-205.
14. Owayed AF, Campbell DM, Wang EE. - Underlying causes of recurrent pneumonia in children. Arch Pediatr Adolesc Med, 2000, 154(2): 190-194.

15. Maxson RT, Harp S, Jackson RJ, et al. - Delayed gastric emptying in neurologically impaired children with gastroesophageal reflux disease: the role of pyloroplasty. J Pediatr Surg, 1994, 29(6): 726-729.

16. Hrabovsky EE, Mullet MD. - Gastroesophageal reflux and the premature infant. J Pediatr Surg, 1986, 21(7): 583-587.

17. Danielson PD, Emmens RW. - Esophagogastric disconnection for gastroesophageal reflux in children with severe neurological impairment. J Pediatr Surg, 1999, 34(1): 84-87.

18. Stephen JL. - Pediatric gastroesophageal reflux disease. Gastroenterol Clin North Am, 1990, 19(3): 617629.

19. Jolley SG. Current surgical considerations in gastroesophageal reflux disease in infancy and chidhood. Surg Clin North Am, 1992, 72(6): 1365-1391.

20. Smith CD, Othersen HB, Gogan NJ, et al. - Nissen fundoplication in children with profound neurologic disability. High risks and unmet goals. Ann Surg, 1992, 215(6): 654-659.

Endereço para correspondência:

Dr. Lisieux Eyer de Jesus

Rua Presidente Domiciano, 52/801

Boa Viagem

24210-270 - Niterói - RJ 